\title{
BARIATRIC SURGERY - a health economic perspective of the prescription costs
}

Sílvia Paredes, Miguel Mendes, Maria Lopes Pereira, Aline Fernandes, Fernando Manso, Maia da Costa, Olinda Marques, Marta Alves

Hospital de Braga

\section{Introduction}

\section{Objectives}

-The economic burden of obesity on healthcare systems is increasing.

-Bariatric surgery, despite its effectiveness, is costly and doubts about its affordability have been raised.

- Few studies have assessed outcomes such as drug use and costs after bariatric surgery.
The aim of this study was to:

-evaluate prescription and prescription costs before and 6 months after bariatric surgery;

- estimate the cost-benefit analysis of bariatric surgery from the

Portuguese National Health Service (NHS) perspective.

\section{Methods}

-A retrospective study was conducted reviewing the medical charts of patients submitted to bariatric surgery in 2014.

-Data recorded included patient's age, gender, weight, height, body mass index (BMI), comorbidities (type 2 diabetes mellitus (T2DM), hypertension, dyslipidemia, and sleep apnea) and prescription drugs and its dosages. The number of pills and insulin dose were compared pre and postoperatively as well as the necessity of using continuous positive airway pressure (CPAP) for sleep apnea.

-Because bariatric surgery is entirely paid by the NHS and since treatments used for the described comorbidities are mainly or totally financed by our NHS, savings for the NHS were estimated.

\section{Results}

$\cdot n=72$

• 11 đ(15,3\%), 61 ( $84,7 \%)$

-Age ranged from 23 to 65 , with a mean of $40 \pm 9,3$ years.

- Gastric bypass $-n=17$; Sleeve gastrectomy $-n=55$

-There was a significant reduction in the prevalence of hypertension, dyslipidemia and T2DM.

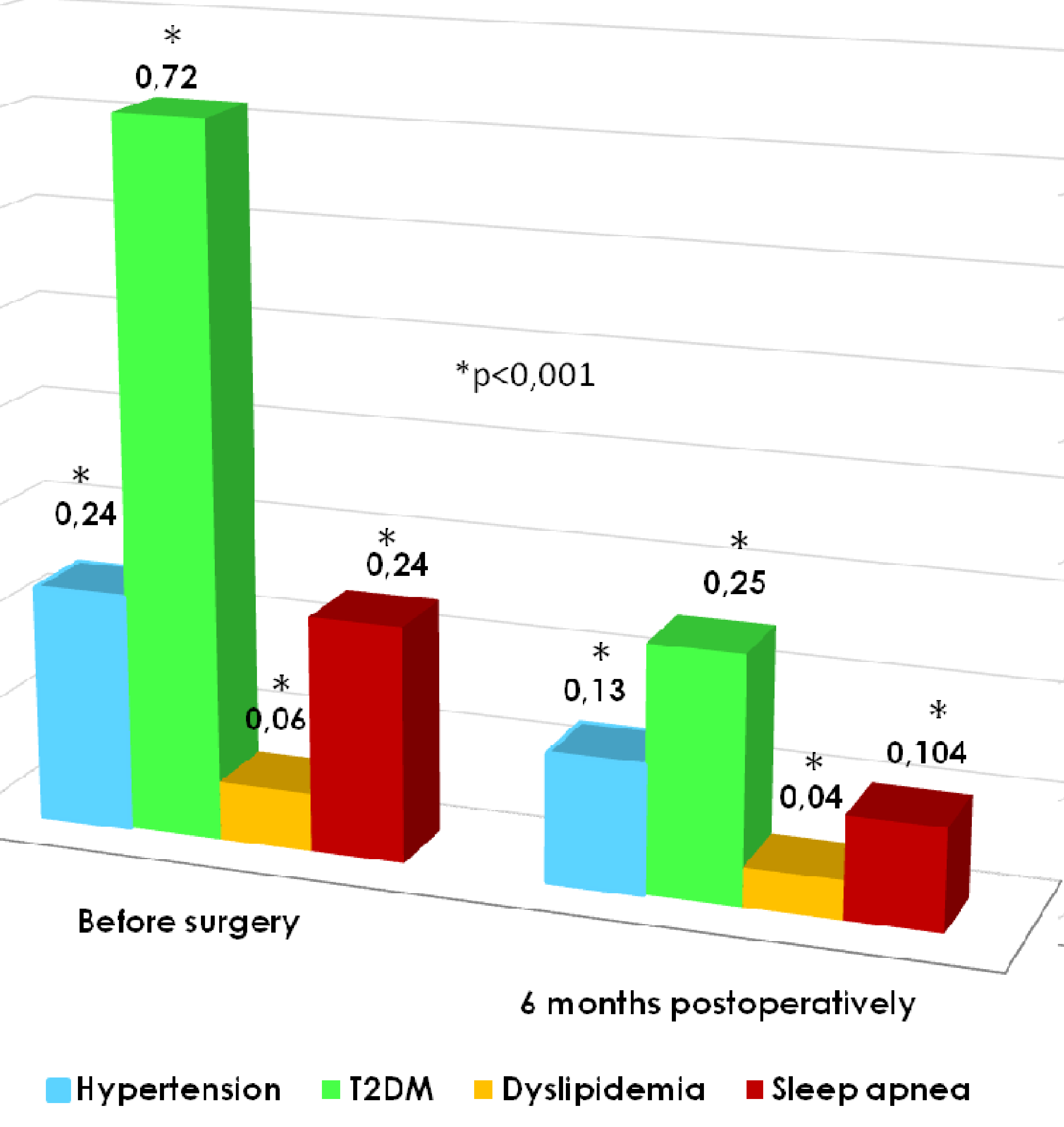

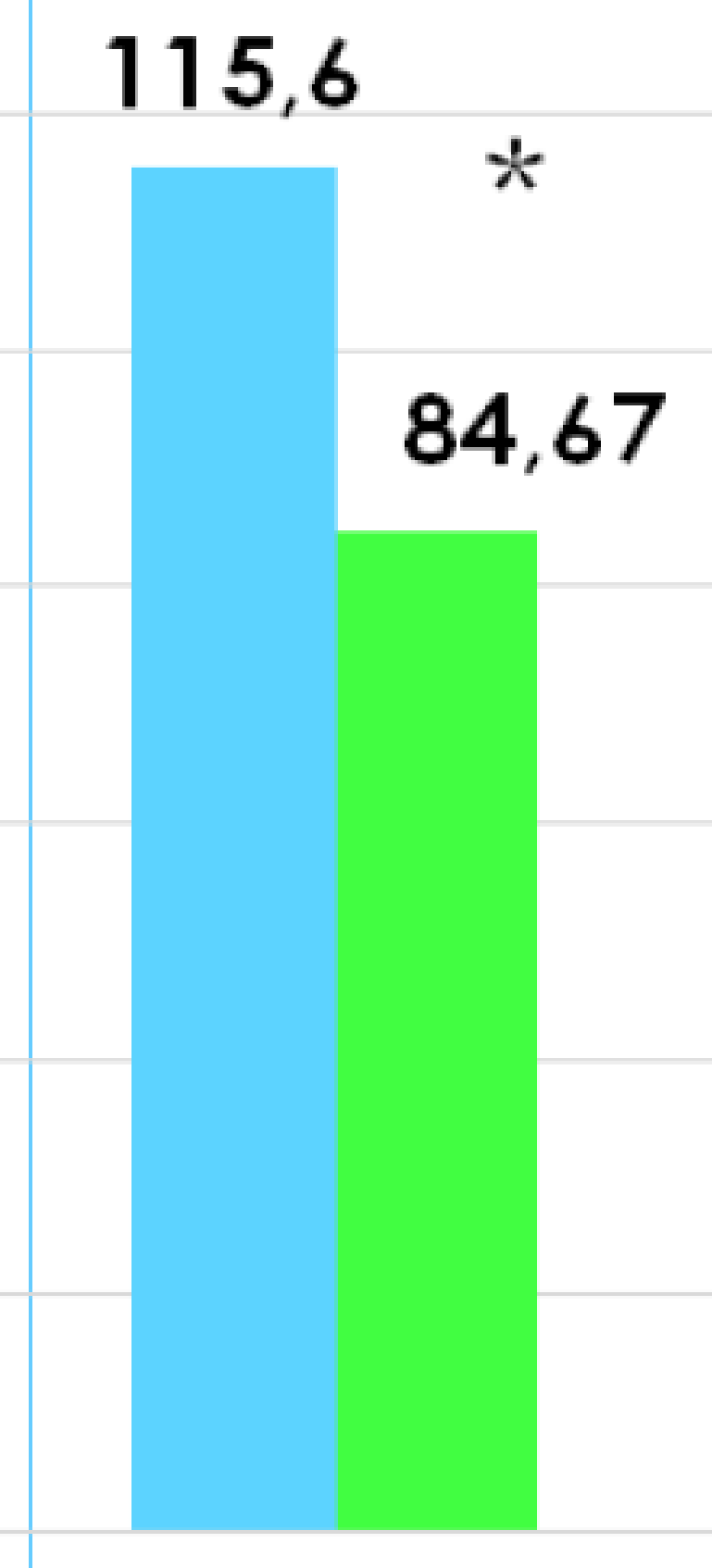

Weight $(\mathrm{Kg})$
84,67 $* p<0,001 ; \S p<0.05$ 